\title{
Adult missing persons: can an investigative framework be generated using behavioral themes?
}

Bonny, E., Almond, L., and Woolnough, Penny

This is the peer reviewed version of the following article:

Bonny, E., Almond, L and Woolnough, P. 2016. Adult missing persons: can an investigative framework be generated using behavioral themes? Journal of Investigative Psychology and Offender Profiling which has been published in final form at doi: https://dx.doi.org/10.1002/iip.1459

This article may be used for non-commercial purposes in accordance with the Wiley Terms and Conditions for Self-Archiving 
Running Head: E. Bonny et al.

Adult missing persons: Can an investigative framework be generated using behavioural themes?

E. Bonny ${ }^{\mathrm{a}}$, L. Almond ${ }^{\mathrm{a}}$ and P. Woolnough ${ }^{\mathrm{b} 1}$

aSchool of Psychology, University of Liverpool, Eleanor Rathbone Building, Bedford

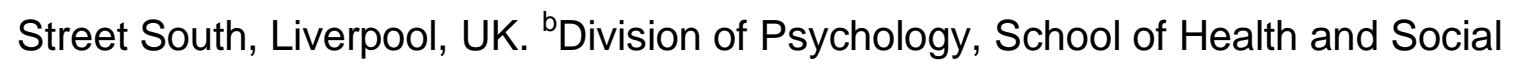

Sciences, Abertay University, Bell Street, Dundee, Scotland.

Emails: e.bonny.07@aberdeen.ac.uk, L.E.Almond@liv.ac.uk

P.Woolnough@abertay.ac.uk

Tel: $0044(0) 1382308638$

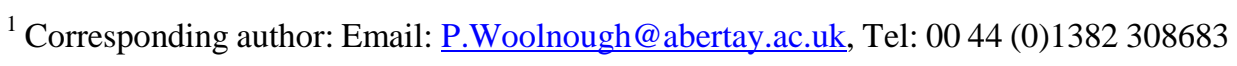




\title{
Adult missing persons: Can an investigative framework be generated using behavioural themes?
}

\begin{abstract}
There is a limited amount of research in the area of missing persons, especially adults. The aim of this research is to expand on the understanding of missing people, by examining adults' behaviours while missing and determining if distinct behavioural themes exist. Based on previous literature it was hypothesised that three behavioural themes will be present; dysfunctional, escape, and unintentional. Thirty-six behaviours were coded from 362 missing person police reports and analysed using smallest space analysis (SSA). This produced a spatial representation of the behaviours, showing three distinct behavioural themes. Seventy percent of the adult missing person reports were classified under one dominant theme, $41 \%$ were 'unintentional', $18 \%$ were 'dysfunctional', and $11 \%$ were 'escape'. The relationship between a missing person's dominant behavioural theme and their assigned risk level and demographic characteristics were also analysed. A significant association was found between the age, occupational status, whether they had any mental health issues, and the risk level assigned to the missing person; and their dominant behavioural theme. The findings are the first step in the development of a standardised checklist for a missing person investigation. This has implications on how practitioners prioritise missing adults, and interventions to prevent individuals from going missing
\end{abstract}

\section{Key words}

Missing persons; adults; behavioural themes; mental health; smallest space analysis. 


\section{Introduction}

It has been estimated that someone is reported missing to police in the UK every 2 minutes (NPIA, 2011). While most return quickly, often within 24 hours, others remain missing for longer and some are never found (Newiss, 1999). Although civil liberties give people the right to go missing, the ambiguous nature of the circumstances and anxiety of those left behind may result in the incident being reported to the police who then face the challenging task of investigating what has happened and attempting to trace the individual.

Highlighting the complexity of the issue, the Association of Chief Police Officers (ACPO) define two categories, 'missing' and 'absent' persons, in their operational guidance. Those 'missing' are defined as "anyone whose whereabouts cannot be established and where the circumstances are out of character or the context suggests the person may be the subject of crime or at risk of harm to themselves or another". Absent, on the other hand, is defined as "a person not at a place where they are expected or required to be" and comprise cases for which there is no apparent risk (although such cases are monitored over periods of time with consideration given to escalating to 'missing' if there is a change to the circumstances that increases the level of risk) (ACPO, 2013 p.5). On this basis, the police are required to risk assess and allocate appropriate resources, both initially and on an on-going basis, to every case reported to them.

During 2011/2012, there were 313,000 missing person reports representing approximately 192,000 people (SOCA, 2013). Given the estimated cost of an average missing person investigation is almost $£ 2500$ (Shalev-Greene \& Pakes, 2012), there is clearly an economic as well as emotional imperative for understanding more about the behaviours of missing people. Missing person incidents are, however, extremely diverse (Newiss, 1999; Biehal, Mitchell, \& Wade, 2003), making accurate risk assessments difficult to develop. For example, while the majority of missing person cases are resolved quickly (within 48 hours) and are traced alive (NPIA, 2011), a small number come to harm, remain 
missing or result in a fatal outcome (about 0.2\%) (Newiss, 1999; NPIA, 2011). The difficulties of predicting such low base-rate events (e.g., missing people who are murdered) is particularly problematic for the police and officers' professional experience and judgement, which is often highly subjective, is heavily relied upon throughout the investigative process (Tarling, \& Burrows, 2004).

Sherman (1998) suggested that police practice, like its medical counterparts, should have its base in scientific evidence about what works best, an approach referred to as evidence-based policing (EBP), using scientifically validated peer reviewed research to guide and evaluate policy and practice (Hoggett \& Stott, 2012). However, missing persons remain a significantly under-researched area, with the limited literature dominated by research into missing people aged under 18 years old, almost to the exclusion of research on adults. The importance of addressing the gap in research is explicitly illustrated by data from the UK Police National Missing Persons Bureau (UKMPB) which shows that nearly $75 \%$ of outstanding missing person reports (classified as those exceeding 14 days) are adults (Newiss, 2005). Furthermore, as duration missing increases the proportion of adult cases increases (Newiss, 2005) and missing adults are more likely to be found dead than children (Home Office, 2011).

Police response to a missing person report first involves the force control room (CR) receiving a phone call from the informant. The operator asks questions to initially risk assess, and either deploy an Emergency Response Team (ERT) unit on an 'Immediate' response for a High Risk or 'Standard' response for a Low or Medium Risk missing person. An ERT Officer then takes the report directly from the informant, by asking investigative questions and searching the missing persons' property, with the occupier's authority. The ERT Officer then completes an electronic missing person report, which involves further police checks. The ERT Officer will use the answers to their initial investigative questions, any knowledge gained from the property search, results from the 
further police checks and their own professional experience and judgement to grade the risk level, they may also consult with the Duty Officer. The report is then reviewed by the Duty Officer who will either confirm the risk level or adjust it recording their justification. (Metropolitan Police Service, 2013).

Consequently, increased understanding of factors which influence missing adults' behaviour would facilitate improved police investigation, risk assessment and response. Developing an investigative framework could help inform officers' narratives of missing (Fyfe, Stevenson and Woolnough, 2014) in a more evidence based manner than at present. This would be similar to Behavioural Investigative Advisors use of thematic models in their investigation of sexual offences (Alison, Goodwill, Almond, van de Heuval, \& Winter, 2010) and the checklist used to assess the risk of contact child sex offences, for individuals found in possession of indecent images of children, KIRAT (CEOP, 2012). ,

Although very little research has specifically considered adult missing persons, three key studies based on missing people of all ages have begun to contribute to our knowledge of missing adults by proposing typologies of missing persons. Utilising 505 police recorded missing person reports, 270 in-depth interviews with family/friends and consultations with 90 organisations with an interest in missing people, an Australian based study proposed three typologies: 'those who leave to gain independence or as a form of rebellion'; 'those that leave to escape adverse consequences'; and 'those that go missing unintentionally', perhaps through miscommunication or an accident (Henderson, Henderson, \& Kiernan, 2000). However, nearly $75 \%$ of this sample represented missing people under the age of 18 so the extent to which these typologies reflect the breadth of missing adult behaviours is questionable.

Similar to Henderson et al. (2000), the first UK based research devised a 'missing continuum' which ranged from intentional to unintentional absences (Biehal, Mitchell, \& Wade, 2003). However, it used data held by the charity National Missing Persons Helpline 
(now called Missing People) which includes cases which are not taken on by police (e.g., long lost relatives/friends rather than someone who has recently gone missing). Consequently, their typology included the categories 'drifted', as well as 'forced to leave' , 'decided to leave' and 'unintentionally absent' which echo those of Henderson et al. (2000) (Biehal, Mitchell, \& Wade, 2003).

Using UK police data, Gibb and Woolnough (2007) conducted the first research to produce behavioural profiles for missing persons based on different ages of children and common mental health disorders, as well as suicide. They produced a booklet of profiling guidance for police officers setting out four operational scenarios or lines of enquiry for police officers to consider: 'lost person' representing someone who has gone missing unintentionally; 'voluntarily missing' where someone has chosen to leave, a 'missing person who may be under the influence of a third party' (e.g., abduction); and 'missing person due to accident, injury or illness'.

Looking across these studies, it is suggested that three main, potentially interrelated, background themes of adult missing persons may exist: 'dysfunctional' (i.e., individuals with mental health issues), 'escape' (i.e., those who decide or feel forced to leave, thus gaining independence and/or escaping adverse consequences) and 'unintentional' (i.e., those who are lost, under the influence of a third party, or reported as a result of a miscommunication or an accident).

\section{'Dysfunctional' missing adults}

Understanding an individual's vulnerability is crucial when a police officer assigns a risk level to a missing person (Gibb \& Woolnough 2007; ACPO, 2013). Indeed, research suggests that the incidence of mental health issues (MHI) among missing adults is as high 
as $80 \%$ and covers a wide range of $\mathrm{MHI}$ including bipolar disorder, depression, psychosis/schizophrenia and dementia (Gibb \& Woolnough, 2007).

In terms of depression, individuals who spend time alone have elevated cortisol levels (Martias, Nicolson \& Freire, 2011) and when these levels occur with low levels of social function it can lead to depression (Tse \& Bond, 2004). Echoing this, missing adults suffering from depression often spend time alone (Gibb \&Woolnough, 2007) walking aimlessly in rural environments and city centres (Stevenson, Parr, Woolnough \& Fyfe, 2013). Anti-depressants and anti-psychotics have been found to be efficient in treating depression (Fournier, et al., 2010) and schizophrenia (Chakos et al., 2011) respectively. Consequently, individuals with $\mathrm{MHI}$, falling within the 'dysfunctional' theme, are likely to be prescribed medication for treatment and may well take this medication while they are missing.

Research suggests that more than $90 \%$ of individuals admitted to hospital because of self-harm have MHI (Haw, Hawton, Houston, \& Townsend, 2001). Furthermore, there is a high rate of suicidal ideation and a higher risk of suicide attempts among individuals with MHI (Harris, \& Barraclough, 1997). Death by suicide is the largest single known cause of death in police missing person cases (Newiss, 2011). Thus, behaviours demonstrated by dysfunctional missing adults may include previous suicide attempts, attending hospital or seeking professional help. Additionally, due to the strong associations between selfharming and substance misuse (Cumming, Covic, \& Murrell, 2006), and between attempted suicide and alcohol intoxication (Powell et al., 2001; Hlady \& Middaugh, 1987), sustaining an injury and/or being under the influence of alcohol while missing could also be exhibited by 'dysfunctional' missing adults.

One of the behavioural symptoms of dementia, a form of $\mathrm{MHI}$, is aimless walking in an attempt to find a safe place (McShane, 2000; Hope, Keene, Fairburn, Jacoby \& McShane, 
1999, Coltharp, Richie, \& Kaas, 1996), this can result in the individual being classified as a missing person. Wandering might, therefore, be indicative of 'dysfunctional' missing behaviour.

\section{Missing adults seeking 'escape'}

Some missing people intentionally decide to leave or remove themselves from a location/situation (Biehal, Mitchell \& Wade, 2003). Research based on interviews with located missing adults found problems with relationships, family and other life stressors are important contributors to an adult's decision to go missing (Biehal, Mitchell, \& Wade, 2003; Stevenson, Parr, Woolnough \& Fyfe, 2013). Traumatic personal events have been found to create panic, guilt and interpersonal conflict within an individual (Lerner, \& Shelton, 2005). Therefore, a high degree of worrying is likely to be associated with an individual trying to escape their problems by going missing. Hence, missing adults may leave in order to escape problems in their lives as a reaction to a crisis or as a form of personal problem-solving (Payne, 1995; Stevenson, Parr, Woolnough \& Fyfe, 2013).

Research has identified two main ways of coping, problem-focused and emotionfocused. Suppressing other competing activities is one type of problem-focused coping (Carver, Scheier \& Weintraub, 1989), which going missing may achieve. Conversely, going missing could be classified as behavioural disengagement, a less useful coping response (Carver, Scheier \&Weintraub, 1989). Behavioural disengagement provides time to think through problems although this may be an unpleasant experience since one is focused on their problems with no potential solutions (Stevenson, Parr, Woolnough \& Fyfe, 2013). Using past memories to improve one's happiness has been found to be effective for both older (Fallot, 1980) and younger adults (Bryant, Smart, \& King, 2005). These findings 
suggest missing adults may go to a familiar, scenic place or a place with happy childhood memories to help improve their mood. They may even use sleep as another method of escaping from their problems (Willey, 1924; Stevenson, Parr, Woolnough \& Fyfe, 2013). Moreover, research has shown links between feeling in danger and sleeping rough or using temporary accommodation as missing adults who are escaping may still feel vulnerable in their new situation and need somewhere to shelter, wash and eat (Biehal, Mitchell, \& Wade, 2003; Stevenson, Parr, Woolnough \& Fyfe, 2013). Deliberate avoidance of the police has also been reported in interviews with intentionally missing adults (Stevenson, Parr, Woolnough \& Fyfe, 2013).

\section{Adults who are 'unintentionally' missing}

Many adults may not perceive themselves to be 'missing' (Rogers, 1986). For example, some may be reported missing due to failure to communicate, a miscommunication or an unintentional incident/accident and have no awareness of the anxiety of others regarding their safety and whereabouts (Henderson, Henderson, \& Kiernan, 2000). Arguably, these 'unintentionally missing' individuals would display the similar behaviours as non-missing people, for instance going on holiday, accessing money, using their mobile phone, all normal everyday behaviours. Logically these individuals would know where they were and after discovering they were reported missing make contact with the police to end any investigation/search. Or they could have suffered an accident or unfortunate event and as a result are not in contact with friends and family.

Table 1. Hypothesised themes 


\section{Aims of the study}

The small amount of existing literature on missing adult suggests that at least three core background themes exist; dysfunctional, escape and unintentional. These three themes represent different experiences and thereby varying psychological processes which may then account for the subsequent behaviour of missing adults. The question therefore arises as to whether characteristics of missing adults, as suggested above, can be identified to reflect these three themes. In conducting the current study, we therefore hypothesised that each of the three background themes would contain a set of thematically similar characteristics that consistently co-occur (see Table 1). Although these three themes have been identified from a review of the small number of published studies, we are not suggesting that they represent mutually exclusive categories, but hypothesise that as they are psychologically distinct the majority of cases will be able to be classified into one dominant theme. If each of these themes contains a subset of distinct characteristics, with one theme of background characteristics being predominant for each individual, then this would have clear implications for the way in which the Police and other agencies differentiate between and respond to the challenge of missing adults in terms of their prevention, protection and provision (Home Office, 2011).

\section{Method}

\section{Procedure}

Information relating to cases of missing adults (aged 18 years and over) reported to Police Scotland (legacy Grampian Police area) between 2009 and 2011 were obtained. In total, 362 cases, only including the most recent missing incident reported if the adult was a frequent missing person, held a completed 'Missing Person Cancellation/Statement Form' which is a structured interview form comprising 54 questions which are completed by a 
police officer with the located missing person in order to act as a debrief, explore vulnerabilities and collect intelligence to use if the person is reported missing again. The form is designed to elicit information pertaining to key elements of the missing person's experiences and behaviours while missing as well as issues associated with their location (e.g., psychological and geographical information). A coding dictionary (see Table 1 and 2) was developed according to the data available from 'Missing Person Cancellation/Statement Form' and 34 behavioural characteristics were coded as either present or absent for each individual. During the coding process two additional behaviours were identified "Visiting a cemetery" and "Travelled aimlessly", resulting in a total of 36 identified adult missing person' behaviours (see Table 1).

As this study relies solely on case files which were not gathered for the purpose of scientific research, the consistency with which information is recorded and the attention to detail in some cases is limited (Canter \& Alison, 2003). Consequently, a dichotomous approach, based on presence/absence was used, as previous research has argued that this approach can be used to ensure maximum clarity and reliability when using records not collected initially for research purposes (Almond, Canter \& Salfati, 2006).

An independent observer was used to investigate reliability of the data coding across a separate sample of 5 'Missing Person Cancellation/Statement Forms' which were randomly selected from those reported to the police in 2012. A Kappa correlation coefficient of $0.97(p<.000)$ was generated suggesting almost perfect agreement reflecting the structured nature of the questions which did not require coder interpretation.

\section{Sample}


The sample consisted of all the completed adult missing person reports received by Police Scotland (legacy Grampian Police area), over the 3 year period from 2009 to 2011, which held a completed 'Missing Person Cancellation/Statement Forms'. Where an individual had been reported missing on more than once during the sampling period, only the most recent missing incident was included, resulting in a total sample of 362 reports. The most recent missing incident was used as repeat missing adults may demonstrate behavioural consistency across episodes which could skew the data.

The age range was from 18 to 90 years old $(\underline{M}=39.28$ years, $\underline{S D}=16.39), 118(33 \%)$ cases related to females and $244(67 \%)$ to males. The sample reflects the findings of previous research that more males (61\%) go missing over the age of 18 than females (39\%) (NPIA, 2011; SOCA, 2013).

Seventy-two percent of the sample were white European, $14 \%$ white Scottish, 5\% from various other ethnic groups and for $9 \%$ ethnicity was unknown. In terms of $\mathrm{MHI}, 56 \%$ were known to have $\mathrm{MHI}$ ranging from depression to learning difficulties. Occupational status was examined, finding that $40 \%$ were unemployed, $38 \%$ employed, $8 \%$ retired, $4 \%$ students, $3 \%$ disable and for $9 \%$ occupational status was unknown.

Each missing person reported to the police is graded as low, medium or high risk. Of the 2152 reports, $46 \%$ were low, $31 \%$ were medium and $23 \%$ were high. The opposite was observed in the sample of 362 , where $27 \%$ were low, $34 \%$ were medium and $39 \%$ were high. Hence, it appears that as risk level increased the likelihood of a Cancellation/Statement Form being completed also increased.

\section{Statistical Analysis}


Using multidimensional scaling procedures, used in previous research (Canter et al. 2003; Almond, Canter \& Salfati, 2006), the present study aimed to investigate the behaviours of missing adults. The data was analysed using the Smallest Scale Analysis (SSA) whose primary assumption is that any underlying structure can be readily appreciated by examining the relationship each variable has with every other variable (Lingoes, 1973). A measure of association is calculated that indicates the degree of co-occurrence between each behaviour exhibited by missing adults with every other missing adult behaviour. Jaccard's coefficient has previously been regarded as the most appropriate measure of association to use for the analysis of police data, as there is a possibility that variables may not have been recorded even though they were perhaps present (Canter et al, 2003). These associations are then rank ordered and represented as a ranked distance in an abstract 'space'. This visual representation was created such that the higher the correlation between any two given behaviours, the closer they will appear on the spatial plot (Guttman, 1968). The resulting patterns can then be examined allowing for the underlying structure of the missing adult behaviours to be identified, and thematic differentiation can then be defined.

\section{Results}

A SSA was carried out on 36 behaviours across 362 missing adults. The three dimensional SSA produced a coefficient of alienation of 0.20 , suggesting a good fit between the SSA plot and the original association matrix (Canter \& Heritage, 1990). Figure 1 represents vectors 1 and 3 of a three dimensional space. Each point within the space represents a behaviour exhibited by the missing adult. The closer any two points are, within the plot, the more likely they are to have occurred together.

Figure 1. SSA thematic analysis of behaviours 
The aim of the study was to examine the extent to which missing adults' behaviours could be classified into three distinct themes. Figure 1 demonstrates how the co-occurrence of the behaviours within the sample can be divided into three thematically similar subgroups made up of a subset of co-occurring behaviours. The behaviours "Going to a familiar place" (81\%) and "Travelling on foot" (62\%) represent the core behaviours displayed by the majority of missing adults; therefore they are not attributable to just one theme.

Table 2 shows the behaviours within each theme and the corresponding percentage frequencies. The highest behaviour in the 'Dysfunction' theme was being "Alone" (49\%) and "Under the influence" of alcohol and/or drugs (37\%); in the 'Escape' theme it was using "Personal Transport" (31\%) and in the 'Unintentional' theme they were "Knowing where intended to go" (60\%) and using "Personal accommodation" (41\%).

Table 2: Behavioural Composition

\section{Dysfunctional}

Ten behaviours exhibited by missing adults can be seen in Figure 1 located at the top left of the plot. The behaviours indicate a vulnerable individual with $\mathrm{MHI}$ or addiction problems and these issues have contributed to them becoming a missing person. These behaviours include: "Taking medical drugs", "Considering, thinking or talking about suicide", "Attempting suicide", "Being under the influence of alcohol or drugs at the time of going missing" and 'Sustaining an injury". The behaviours "Being alone" and "Walking aimlessly" indicate the individual's social, isolation whilst "Seeking professional help" and "Attending 
or leaving a hospital" suggest they may contact relevant agencies. An extra variable also within this region which was found during data coding, was "Attending a cemetery". This potentially indicates the individuals morbid thought processes.

\section{Escape}

Twelve behaviours seen in Figure 1 at the bottom left of the plot reflect an individual who is trying to cope with life stressors through escaping, indicated by the behaviours "Worrying", "Feeling in danger", and "Intentionally avoiding the police". These individuals attempt to solve their problems by "Sleeping", "Thinking or clearing their head", "Attending a scenic place" or "Attending a place of childhood memories". They gain physical distance from their problems by "Travelling aimlessly" (the second variable added during coding) using "Personal transport", "Emergency accommodation" and "Commercial accommodation" resulting in some individuals "Travelling to another county or further".

\section{Unintentional}

The remaining twelve behaviours can be seen in Figure 1 located on the right hand side of the plot. These behaviours reflect an individual unintentionally missing. A relative or friend may have overreacted or a miscommunication may have occurred. Indicated by the behaviours "Knowing where they intend to go", "Going on holiday", "Recreational activities", "Going to a drinking establishment", "Drinking alcohol" and "Experiencing an unfortunate event". These individuals are not concerned about leaving traces of their whereabouts indicated by them "Using a mobile", "Accessing money", "Using public transport" and "Attending personal accommodation". Once they realised they were reported missing, many "Attended a police station" and "Sort personal help". 
Kuder-Richardson 20 (K-R 20) coefficients were calculated in order to provide an index of internal reliability. The K-R 20 is equivalent to Cronbach's Alpha coefficient but can be used with dichotomous data. The Dysfunctional theme was .567, the escape theme was .516 and the unintentional theme was .518 ; these values are reasonable considering the data was not collected for research purposes. They also equate with other published models which have used this coefficient (Canter et al, 2003 \& Almond et al. 2006). These coefficients are not sufficiently high enough to demonstrate evidence of a scale, however they are sufficiently high enough to indicate that the themes are meaningful and coherent (Almond, et al. 2006).

\section{Classifying Missing Adults in Terms of a Dominant Behavioural Theme}

The current study proposed that the behaviours displayed by a missing adult would reflect one dominant behavioural theme. An individual may display behaviours from more than one theme but as they are psychologically distinct, it was hypothesised that the majority would be able to be assigned to one dominant theme. To identify dominant themes of behaviour within the sample, the same criteria used by Almond, Canter and Salfati (2006) was adopted. The percentage of occurrence of the variables in the dominant theme had to be greater than the sum of the percentage occurrence for the other two themes. If the sum of the two highest percentages of the three behavioural themes was greater than that of triple the lowest, the case was assigned as a hybrid of the two highest behavioural themes. If not it was deemed unclassifiable since no themes were dominant.

Using this method $70 \%$ of the 362 missing adults were able to be classified into a dominant theme; $41 \%$ were 'Unintentional', $18 \%$ were 'Dysfunctional' and $11 \%$ were 'Escape'. A further 8\% were classified as 'Escape-Dysfunctional', 7\% were 'UnintentionalEscape', $6 \%$ were 'Unintentional-Dysfunctional' and $9 \%$ were unclassifiable. 
Analysis of dominant theme and demographic characteristics

Chi-squared tests were conducted to determine if the gender, age, ethnicity, occupational status, risk level and mental health of missing adults were distributed differently across the themes. Hybrid cases were excluded from this analysis. No significant relationship was found between gender or ethnicity and the themes. Therefore, the gender and ethnicity of a missing adult has no indication of the type of missing person.

Table 3. Dominant theme

A significant association was found between the age of missing adults and their dominant behavioural theme: $x 2(4)=24.233 ; p<.001$. Young missing adults aged 30 years and below were more likely to go missing 'Unintentionally', whereas those aged 31-60 years old were more likely to go missing as a means of 'Escape', followed by the theme 'Dysfunctional'. Adults over 60 years old were more likely to go missing due to 'Dysfunctional' reasons, followed by 'Escape'. Cohen's effect size w was used to determine the strength of the association (Volker, 2006) and this association was found to be a medium effect: $w=0.308$.

A further chi-squared test was conducted on the sample excluding cases where the occupational status was unknown. A significant association was found between the missing adults' occupational status and their dominant theme: $x 2(8)=18.702 ; p<.05$. Unemployed missing adults were more likely to be classed as 'Dysfunctional' or 'Unintentional' and less likely to go missing trying to 'Escape' problems compared to 
employed missing adults. This association was found to be a nearing a medium effect: $w=$ 0.287.

A significant association was found between whether a missing adult had $\mathrm{MHI}$ and their dominant theme: $\mathrm{X} 2(2)=8.145 ; \mathrm{p}<.05$. Missing adults with MHI were more likely to be of the behavioural theme 'Dysfunctional', whereas missing adults without MHI were more likely to be 'Unintentionally' missing. This association was found to be nearing a medium effect: $\mathrm{w}=0.253$.

Finally when examining the association between the risk level assigned to a case and their dominant theme a significant relationship was found: $x 2(4)=29.637 ; p<.001$ whereby missing adults graded as high risk were more likely to be of the themes 'Escape' and 'Dysfunctional'. In contrast, missing adults graded low or medium risk were more likely to be missing 'Unintentionally'. This association was found to be a medium effect: $w=$ 0.341.

\section{Discussion}

Existing literature suggests the existence of three main background themes of adult missing persons (Henderson, Henderson, \& Kiernan, 2000; Biehal, Mitchell, \& Wade, 2003; Gibb, \& Woolnough, 2007); dysfunctional, escape and unintentional. Results of the current study show a heterogeneous sample (since the majority of the behaviours have low to medium frequencies); therefore suggesting that different sub-groups are likely to exist. Three behavioural themes were uncovered in the SSA plot (Figure 1), with the majority of behaviours falling within one of the predicted behavioural themes, as hypothesised. Interestingly, however, the behaviour 'tried to get help' fell within the theme 'unintentional' instead of 'escape' as predicted (Table 1). The second hypothesis was also supported as $70 \%$ of the cases were able to be classified under one dominant behavioural theme. Three behaviours are present in over $60 \%$ of the cases, these being "Knowing 
where they intend to go", "Going to a familiar place" and "Travelling on foot". These would be regarded as the primary behaviours, which the majority of the sample exhibited. Unlike the low frequency behaviours, they do not distinguish between the themes.

The first Home Office cross government missing person strategy, published in 2011, outlines three objectives: prevention, protection and provision (Home, Office, 2011). The first objective, concerns reducing the amount of people who go missing in the first place, through a better understanding of missing people. Secondly, to protect missing adults who are at risk, the Home Office (2011) states that response to a missing person must be specific to the missing individual. The current findings have aided in the understanding of missing adults as well as helping to identify two different types of missing adults, escape and dysfunctional, which appear to represent vulnerable individuals at a higher risk of coming to harm. Different responses, therefore, would be appropriate and more effective for these two themes. Further analysis showed different reasons for going missing reflecting the two different behavioural themes. Problems with family relationships, work, and finances were more likely to reflect an 'escaping' missing adult, whereas illness, drug and alcohol addiction a 'dysfunctional' missing person.

Police Officers could utilise these findings to help focus their initial investigative questions with the informant and wider family/friends. Similar to Police Officers' use of the KIRAT checklist for assessing the risk of contact child sex offences for suspects found in possession of indecent images of children (CEOP, 2012) the current findings could be the first step in the development of a standardised checklist for a missing person investigationfor which it is mandatory to ask all questions even if the Officer does not deem them relevant, this could be beneficial in a missing person investigation, as it would prevent the risk assessment relying as heavily on an Officers' professional experience and judgement, which is often highly subjective. The questions, designed around the behaviours uncovered in the current research, could be divided into four different sections 
to reflect the three behavioural themes and a fourth section including questions relating to low-base rate events (e.g., being a victim of crime). This would potentially generate more information in order to make a more informed risk assessment, by both the ERT Officer and the Duty Officer, and therefore assist in determining the appropriate level of police response.

Biehal, Mitchell and Wade's (2003) 'missing continuum' proposed the existence of individual's going missing unintentionally. The current results support this since $41 \%$ of the cases were classified under 'unintentional'. This has serious implications because it suggests nearly half of missing person cases could be an unnecessary use of police resources. Supporting this implication, our findings showed cases classified under the 'unintentional' theme were less likely to be high risk. Nonetheless, someone has been sufficiently concerned for someone's welfare that they have called the police. In terms of Individuals more likely to be missing unintentionally, these were most commonly: those aged 18-30 years old, students, people with no $\mathrm{MHI}$ and no existing problems in their lives. Hence, early interventions such as educational work would be beneficial to prevent these individuals being reported missing. Specifically, by emphasising the importance of communication, outlining how worrying can escalate, and informing individuals of the amount of money and resources spent on missing people, the number of unintentional missing adults could, potentially, be reduced. Exploring this issue, through the use of questionnaires or in-depth interviews with individuals who report someone missing who is subsequently found to be unintentionally missing, would be beneficial.

Interestingly, an unexpected behaviour was present in the 'unintentional' behavioural theme, "tried to get personal help". However, on closer inspection this behaviour does fit with this theme as it relates to individuals 'attending a police station' and seeking 'personal help' once they became aware that they had been reported missing. 
The other end of the 'missing continuum' relates to those individuals who go missing intentionally (Biehal, Mitchell, \& Wade, 2003). Previous research suggested the existence of subgroups of intentional missing people; dysfunctional and escape (Henderson, Henderson, \& Keirnan, 2000; Gibb, \& Woolnough, 2007). The present findings support this; since it found $18 \%$ of the cases were classified as 'dysfunctional' and $11 \%$ as 'escape'. These individuals were more likely to be graded high risk, and therefore should be the focus for early interventions, by means of two different approaches.

Programmes have been developed recently which have relevance for missing people who fall within the behavioural theme 'dysfunctional', specifically in regard to mental health disorders. In England, the Dementia Commissioning Pack, as well as 'no health without mental health', and in Wales the National Dementia Vision (Home Office, 2011). This shows an encouraging step by the government to tackle these serious issues within the area of missing people. However, they are geared towards all people with $\mathrm{MHI}$, not all of whom may go missing.

The current study has uncovered multiple behaviours related specifically to 'dysfunctional' missing adults. Social services could use these behaviours to create risk assessment tool to predict risk of going missing. For example, if an individual known to social services has MHI, takes medical drugs, has suicidal ideation, has attempted suicide, often drinks or uses other drugs, spends time in hospital, and has been known to walk around aimlessly usually alone, they would be deemed high risk of becoming a missing person. The development of tailored interventions might prevent this from happening, thus allowing police resources to be allocated to other pressing investigations.

Further analysis found individuals likely to fall under the 'dysfunctional' theme were over the age of 60 and retired. Individuals with MHI were also more likely to fall under this theme. Thus, further areas which should be targeted for early interventions are retirement homes and community centres. 
The findings of this research suggest a strong influence of $\mathrm{MHI}$ and demonstrate the need for strategies specific to individuals dealing with difficult situations in their lives. The government has recently established the Ministerial Working Group on Homelessness, as well as the 'Ten Year Homelessness Plan for Wales 2009-2019'. However, these only target one of the behaviours exhibited by individuals in the behavioural theme 'escape'. Consequently, additional strategies, by further examining the 'dysfunctional' behaviour theme, need to be established.

Missing adults aged 31-60 years largely fell within the 'escape' theme. These individuals were more likely to have problems with family relationships, work and finances, and therefore went missing as a way to cope. This supports research regarding coping mechanisms (Carver, Scheier, \& Weimtraub, 1989). These findings highlight that workplace interventions could be beneficial, especially as missing adults who fall into the 'escape' theme were more likely to be employed. Workplace interventions for $\mathrm{MHI}$ have been found to be successful in reducing the total symptom load of depression (Ahola, Vuori, Toppinen-Tanner, Mutanen \& Honkonen, 2012). Thus if managers received training to gain a greater knowledge of the likely behaviours exhibited by missing adults, they would be more likely to provide support and interventions, to prevent them from going missing as a way of coping or due to $\mathrm{MHI}$.

Interestingly, when examining the SSA plot (Figure 1), there appears to be a gap where no variables are present between the 'dysfunctional' and 'unintentional' theme. Previous research has proposed that gaps in SSA plots are actually quite meaningful (Canter, Hughes, \& Kirby, 1998). An explanation for the gap could be that it represents low baserate events and, therefore, behaviours exhibited by missing adults found deceased, irrespective of whether this outcome was intentional or unintentional. While further research on this issue would be difficult to conduct, analysis of police sudden death reports or in-depth interviews with family and friends could help to shed some light on this. 
A limitation of the present research may be related to the sample of 362 missing person reports which contained 'Missing Person - Cancellation/Statement Forms'. Unfortunately, the sample is biased due to a relatively low completion rate for cancellation forms with located missing adults (16.8\%) meaning that it may not be appropriate to generalise the findings to all located missing adults. Also, the sample and findings do not take account of those for whom a cancellation form could not be completed because they were not located (i.e., remained missing) or were found dead. Hence, there may be a bias towards less serious cases. However, $39 \%$ of the final sample was recorded as high risk, $34 \%$ medium and the remaining $27 \%$ low. This contrasts with the risk assessment across the total 2152 cases of missing adults from 2009 to 2011 where $23 \%$ were high, $31 \%$ medium and $46 \%$ low risk, suggesting that, for those located, there is an emphasis on more serious cases. This is a broader issue which has previously been highlighted when police data is used for research purposes (Canter, \& Alison, 2003). Previous research has shown police officers assign risk levels through determining the individual's level of vulnerability. The young, elderly, mentally infirm and drug-dependent are deemed to be vulnerable (Newiss, 1999), this indicates that high risk cases would mainly reflect the 'dysfunctional' theme. Thus, within the present study the theme 'dysfunctional' would be overrepresented whereas the 'unintentional' theme may be underrepresented since it more likely represent low risk cases.

Previous research found that $80 \%$ of missing adults had MHI (Gibb, \& Woolnough, 2007; Home Office, 2011). In the current study only $56 \%$ reported some form of MHI. This may be because the current study was based on analysis of the 'Missing Person Cancellation/Statement Forms' only, whereas Gibb and Woolnough (2007) utilised the full police report (which includes the Missing Person - Cancellation/Statement Form') which yields more data. Consequently, future SSA work should consider using the full police missing person report rather than just the 'Missing Person - Cancellation/Statement Form'. 
The present study used police data that was not originally collected for the purpose of conducting research. While it was collected using a semi-structured questionnaire designed to acquire knowledge about a missing persons experience and behaviours, it was administered by a police officer in an interview setting and not a trained researcher. Furthermore, missing adults may lie (Canter, \& Alison, 2003), especially when asked questions which may incriminate or reflect badly on them. Therefore, social desirability bias may affect the data's validity. Additionally, quality and validity of police data can sometimes be questionable. Previous research has found that even an offender's gender can be recorded inaccurately (Farrington, \& Lambert, 2000). This may explain why a significant association between a missing person's ethnicity and their dominant behaviour theme was not present. However, while these limitations should be borne in mind, police records have been utilised by many researchers in the field of investigative psychology to great effect (Canter, Bennell, Alison, \& Reddy, 2003; Salfati, \& Canter, 1999). In this vein, the current research successfully used police data to support both hypotheses.

This paper presents the first empirically tested thematic analysis of missing peoples' behaviour. The SSA analysis uncovered three behavioural themes; dysfunctional, escape and unintentional, and it was able to successfully classify $70 \%$ of the missing adults under one dominant theme. These typologies are therefore based on statistical analyses rather than descriptive analysis and provide an objective investigative framework to guide practitioners, officers and future research. This has implications for the prevention, for interventions in workplace, educational settings and retirement homes, and protection of missing persons, through the potential development of a more standardised risk assessment protocol that can be utilised by the police and wider agencies responsible for the safeguarding of vulnerable adults (Home Office, 2011). 


\section{References}

Ahola, K., Vuori, J., Toppinen-Tanner, S., Mutanen, P., \& Honkonen, T. (2012). Resourceenhancing group intervention against depression at workplace: who benefits? A randomised controlled study with a 7-month follow-up. Occupational and Environmental Medicine, 69(12), 870-6.

Alison, L., Goodwill, A., Almond, L. Van de Heuval, C. \& Winter, J. (2010). Pragmatic solutions to offender profiling and behavioural investigative advise. Legal and Criminological Psychology, 15, 115-132.

Almond, L., Canter, D., \& Salfati, G. (2006). Youths Who Sexually Harm: A Multivariate Model of characteristics. Journal of Sexual Aggression, 12(2), 97-114.

Association of Chief Police Officers (2013). Interim Guidance on The Management Recording and Investigation of Missing Persons. London: College of Policing.

Biehal, N., Mitchell, F., \& Wade, J. (2003). Lost from view. Missing persons in the UK. UK, Bristol: The Policy Press.

Bryant, F., Smart, C., \& King, S. (2005). Using the past to enhance the present: Boosting happiness through positive reminiscence. Journal of Happiness Studies, 6, 227-260.

Canter, D., Bennell, C., Alison, L. \& Reddy, S., (2003). Differentiating Sex Offences: A Behaviourally Based Thematic Classification of Stranger Rapes, Behavioural Sciences \& Law, 21, 157-174. 
Canter, D., \& Alison, L. J. (2003). Converting Evidence Into Data: The Use of Law Enforcement Archives As Unobtrusive Measurement. The Qualitative Report, 8(2), 151-176.

Canter, D., Hughes, D., \& Kirby, S. (1998). Paedophilia: Pathology, criminality, or both? The development of a multivariate model of offence behaviour in child sexual abuse. The Journal of Forensic Psychiatry, 9, 532-555.

Canter, D. \& Heritage, R. (1990). A multivariate model of sexual offence behaviour: Developments in 'offender profiling'. The Journal of Forensic Psychiatry, 1, 185-212.

Carver, C., Scheier, M., \& Weintraub, J. (1989). Assessing coping strategies: A theoretically based approach. Journal of Personality and Social Psychology, 56, 267283.

Child Exploitation and Online Protection (2012). A picture of abuse: A thematic assessment of the risk contact child sexual abuse posed by those who possess indecent images of children. Accessed from:

www.ceop.police.uk/Documents/ceopdocs/CEOP\%20IIOCTA\%20Executive\%20Sum mary.pdf

Chakos, M., Patel, J., Rosenheck, R., Glick, I., Hammer, M., Tapp, A., \& Miller, A. (2011). Concomitant Psychotropic Medication Use During Treatment of Schizophrenia Patients: Longitudinal Results from the CATIE Study. Clinical Schizophrenia \& Related Psychoses, 5(3), 124-134. 
Coltharp, W. Jr., Richie, M., \& Kaas, M.(1996). Wandering. Journal of Gerontological Nursing, 22, 5-10.

Cumming, S., Covic, T., \& Murrell, E. (2006). Deliberate self-harm: Have we scratched the surface? Behaviour Change, 23, 186-199.

Fallot, R. (1980). The impact on mood on verbal reminiscing in later adulthood. International Journal of aging and Human Development, 10, 385-400.

Farrington, D., \& Lambert, S. (2000). Statistical approaches to offender profiling. In D. V. Canter \& L. J. Alison (Eds.), Profiling property crimes (pp. 233-274). Aldershot: Ashgate.

Fournier, J., DeRubeis, R., Hollen, S., Dimidjian, S., Amsterdam, J., Shelton, R., \& Fawcett, J. (2010). Antidepressant Drug Effects and Depression Severity. A PatientLevel Meta-analysis. Journal of American Medical Association, 303(1), 49-53.

Fyfe, N., Stevenson, O. and Woolnough, P. (2014). Missing persons: the processes and challenges of police investigation. Policing and Society. ISSN 1043-9463 (doi:10.1080/10439463.2014.881812) (Early Online Publication).

Gibb, G. J. \& Woolnough, P. (2007). Missing Persons: Understanding, Planning, Responding. UK, Aberdeen: Grampian Police.

Guttman, L. (1968). A general nonmetric technique for finding the smallest coordinate space for configuration of points. Psychometrika, 33, 465-506. 
Halek, M. \& Bartholomeyczik, S. (2011). Description of the behaviour of wandering in people with dementia living in nursing homes - a review of the literature. Scandinavian Journal of Caring Sciences, 26, 404-413.

Harris, E., \& Barraclough, B. (1997). Suicide as an outcome for mental disorders. British Journal of Psychiatry, 170, 205-228.

Haw, C., Hawton, K., Houston, K., \& Townsend, E. (2001). Psychiatric and personality disorders in deliberate self-harm patients. The British Journal of Psychiatry, 178, 4854.

Henderson, M., Henderson, P., \& Kiernan, C. (2000). Missing persons: Incidences, issues and impacts. Australian Institute of Criminology: Trends and Issues in Crime and Criminal Justice, 144, 1-6.

Hlady, W., \& Middaugh, J. (1987). The epidemiology of suicide in Alaska, 1983-1984. Alaska Medicine, 29, 158-164.

Hoggett, J., \& Stott, C. (2012). Post G20: The challenge of change, implementing evidence-based public order policing. Journal of Investigative Psychology and Offender Profiling, 9(2), 174-183.

Home Office (2011). Missing Children and Adults: A Cross Government Strategy. London: Home Office. 
Hope, T., Keene, J., Fairburn, C., Jacoby, R., \& McShane, R. (1999). Natural history of behavioural changes and psychiatric symptoms in Alzheimer's disease. British Journal of Psychiatry, 174, 39-44.

Lerner, M., \& Shelton, R. (2005). Comprehensive acute traumatic stress management. NY, Commack: American Academy of Experts in Traumatic Stress.

Lingoes, J. (1973).The Guttman Lingoes Non-metric program Series. Ann Arbor, Ml: Mathesis.

Martias, G., Nicolson, N., \& Freire, T. (2011). Solitude and cortisol: Associations with state and trait affect in daily life. Biological Psychology, 86, 314-319.

Metropolitan Police Service (2013). Missing Persons Toolkit - Primary Investigation Frontline Checklist (Standard Operating Procedure). London: MPS

McShane, R. (2000). What Are the Syndromes of Behavioural and Psychological Symptoms of Dementia? International Psychogeriatric, 12, 147-153.

Newiss, G. (1999). Missing presumed...? The police response to missing persons. Police Research Series, No 114. London: Home Office.

Newiss, G. (2005). A Study of the Characteristics of Outstanding Missing Persons: Implications for the Development of Police Risk Assessment. Policing \& Society, 15(2), 212-225. 
Newiss, G. (2011). Going missing on a night out: men found dead in water. London, Missing People.

National Policing Improvement Agency (2011). Missing persons: Data and analysis 2010/2011. London: NPIA.

Serious Organised Crime Agency (2013). Missing persons: Data and analysis 2011/2012. London: SOCA.

Payne, M. (1995). Understanding 'Going Missing': issues for social work and social services. British Journal of Social Work, 25, 333-348.

Powell, K., Kresnow, M-J., Mercy, J, Potter, L., Swann, A., Frankowski, R., Lee, R., \& Bayer, T. (2001). Alcohol Consumption and Nearly Lethal Suicide Attempts. Suicide, and Life-Threatening Behaviour, 32, 30-41.

Richards, L., Letchford, S., \& Stratton, S. (2008). Policing Domestic Violence. Oxford: Oxford University Press.

Rogers, C. (1986). Tracing missing persons: An introduction to agencies methods and sources in England and Wales. Manchester: Manchester University Press.

Salfati, C, \& Canter, D. (1999). Differentiating Stranger Murders: Profiling Offender Characteristics from Behavioral Styles. Behavioral Sciences and Law, 17, 391-406.

Shalev-Greene, K., \& Pakes, F. (2012). Establishing the Cost of Missing Person Investigations. UK, Portsmouth: The Centre of the Study of Missing Persons. 
Sherman, L. (1998). Evidence Based Policing. Washington, DC: Police Foundation. Available at www.policefoundation.org/pdf/Sherman.pdf.

SOCA (2013). Missing Persons: Data and analysis 2011/2012, Serious Organised Crime Agency.

Stevenson, O., Parr, H., Woolnough, P., \& Fyfe, N. (2013). Geographies of Missing People: Processes, Experiences, Responses. University of Glasgow, Glasgow, UK.

Tarling, R., \& Burrows, J. (2004). The nature and outcome of going missing: the challenge of developing effective risk assessment procedures. International Journal of Police Science \& Management, 6(1), 16-26.

Tse, W., \& Bond, A. (2004). Relationship between baseline cortisol, social functioning and depression: a mediation analysis. Psychiatry Research, 126, 197-201.

Volker, M. (2006). Reporting Effect Size Estimates in School Psychology Research. Psychology in the Schools, 43(6), 653-672.

Willey, M. (1924). Sleep as an Escape Mechanism. The Psychoanalytic Review, 11, 181183. 


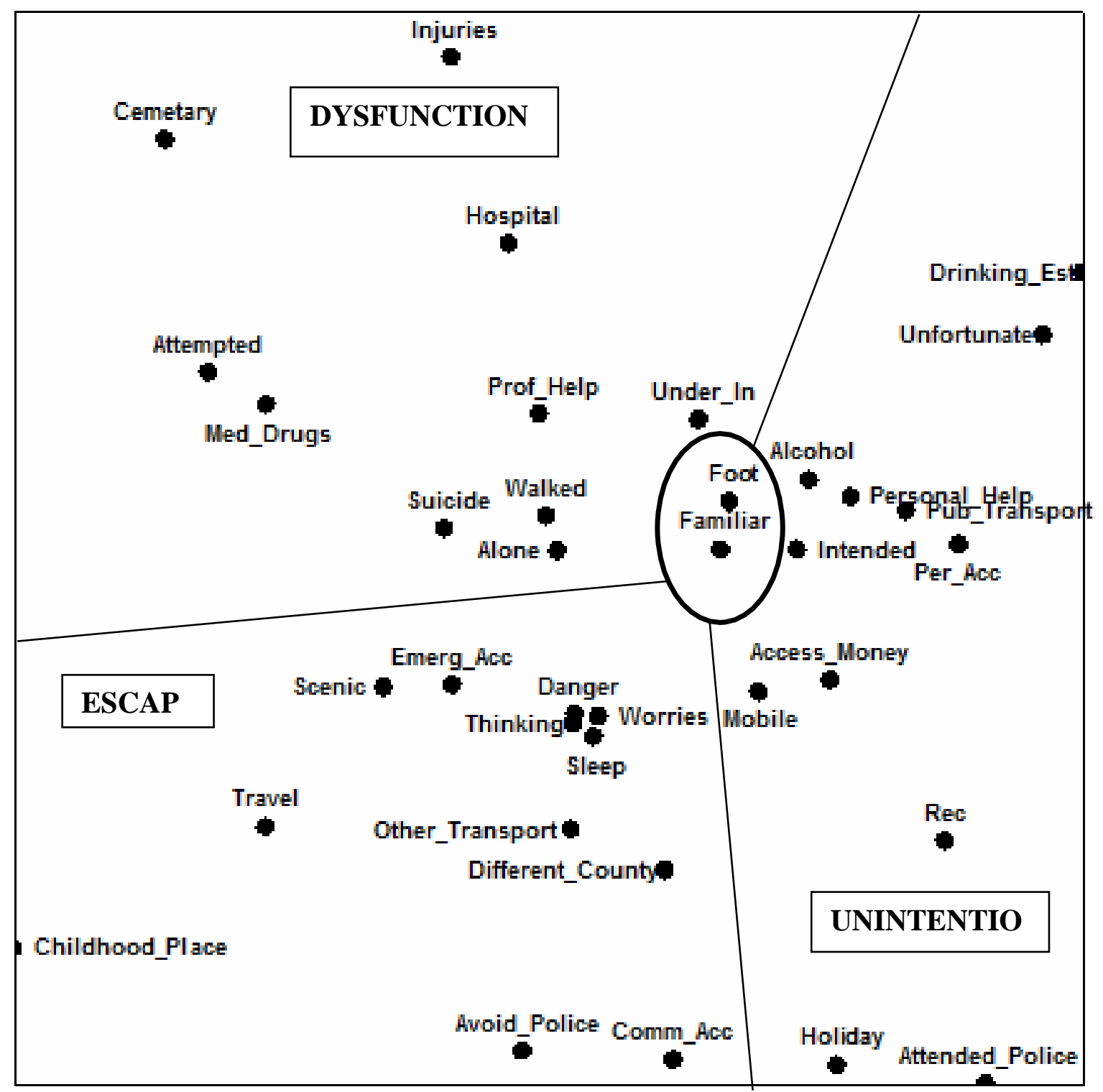

Figure 1. 1by 3 projection of a three dimensional Smallest Space Analysis (SSA)of 36 behaviours displayed by 362 Missing Adults. The SSA indicates the different themes in behaviour. Variable labels are brief summaries, full definitions can be found in Table 2. 
Table 1. Hypothesised behavioural themes.

\begin{tabular}{|c|c|c|}
\hline \multicolumn{3}{|c|}{ Behavioural Themes } \\
\hline Dysfunctional & Escape & Unintentional \\
\hline Travelling on Foot & Familiar place & Access Money \\
\hline Alone & Thinking or Clearing Mind & Used Mobile \\
\hline Medical Drugs & Place of Childhood Memories & Consumed Alcohol \\
\hline Hospital & Scenic Place & Attended Police Station \\
\hline Suicide Thoughts & Personal Help & Knew where intended to go \\
\hline Attempted Suicide & Sleeping & Public Transport \\
\hline Professional Help & Worries & Personal Accommodation \\
\hline Injuries & Feeling in Danger & Drinking Establishment \\
\hline Under Influence & Emergency Accommodation & Unfortunate Incidents \\
\hline Walked Aimless & Commercial Accommodation & Recreational Activities \\
\hline Visiting a Cemetery & Avoid Police & Holiday \\
\hline & Personal Transport & \\
\hline & Different County & \\
\hline & Travelled Aimlessly & \\
\hline
\end{tabular}


Table 2. Behavioural composition of themes

\begin{tabular}{|c|c|c|c|}
\hline Theme & Name & Explanation & Freq \% \\
\hline \multirow[t]{2}{*}{ Core Variables } & Familiar & Going to a familiar place & 81 \\
\hline & Foot & Travelled on foot & 62 \\
\hline \multirow[t]{10}{*}{ Dysfunctional } & Alone & Was unaccompanied while missing & 49 \\
\hline & Under_In & $\begin{array}{l}\text { Under the influence at time of going } \\
\text { missing }\end{array}$ & 37 \\
\hline & Walked & Walked around as an activity & 25 \\
\hline & Suicide & $\begin{array}{l}\text { Considered, talked about, and/or thought } \\
\text { about suicide }\end{array}$ & 22 \\
\hline & Hospital & $\begin{array}{l}\text { Left attended hospital, or rehabilitation } \\
\text { unit. }\end{array}$ & 19 \\
\hline & Prof_Help & $\begin{array}{l}\text { Tried to get help from telephone helpline, } \\
\text { advice/counselling agency, housing } \\
\text { agency, social worker, doctor/nurse and/or } \\
\text { police }\end{array}$ & 12 \\
\hline & Med_Drugs & Took medical drugs & 11 \\
\hline & Injuries & Sustained injuries & 8 \\
\hline & Attempted & $\begin{array}{l}\text { Attempted suicide (e.g. car exhaust, } \\
\text { overdose, cutting wrists, hanging, and } \\
\text { drowning) }\end{array}$ & 8 \\
\hline & Cemetery & Attended a cemetery. & 3 \\
\hline \multirow[t]{12}{*}{ Escape } & Per_Trans & $\begin{array}{l}\text { Used other transport (eg. car, bicycle, } \\
\text { motorbike and hitched a lift) }\end{array}$ & 31 \\
\hline & Thinking & $\begin{array}{l}\text { Thinking, clearing head, need space. Went } \\
\text { to calm down, relax, chill or somewhere } \\
\text { quiet. }\end{array}$ & 24 \\
\hline & Worries & Worried about returning & 22 \\
\hline & Scenic & Attended a scenic place. & 17 \\
\hline & Emerg_Acc & $\begin{array}{l}\text { Stayed outdoors/slept rough, at social } \\
\text { services accommodation, with someone } \\
\text { they just met or in a vehicle }\end{array}$ & 16 \\
\hline & Different_County & Went to another county/region or further. & 15 \\
\hline & Danger & Felt in danger & 15 \\
\hline & Sleep & Spent time sleeping. & 14 \\
\hline & Travel & $\begin{array}{l}\text { Drove about, sat in car / car park or } \\
\text { travelled around on bus - no destination in } \\
\text { mind. }\end{array}$ & 14 \\
\hline & Avoid_Police & Intentionally avoided the police & 11 \\
\hline & Comm_Acc & $\begin{array}{l}\text { Stayed in a hotel, bed and breakfast or } \\
\text { hostel }\end{array}$ & 9 \\
\hline & Childhood_Place & $\begin{array}{l}\text { Went to place because of childhood } \\
\text { memories. }\end{array}$ & 3 \\
\hline \multirow[t]{5}{*}{ Unintentional } & Intended & Knew where they intended to go & 60 \\
\hline & Per_Acc & $\begin{array}{l}\text { Stayed at home, at a friends or family } \\
\text { house }\end{array}$ & 41 \\
\hline & Alcohol & Consumed alcohol & 41 \\
\hline & Pub_Transport & $\begin{array}{l}\text { Used public transport (e.g. bus, train, plane } \\
\text { and boat) }\end{array}$ & 35 \\
\hline & Mobile & Used a mobile phone & 29 \\
\hline
\end{tabular}


Access_Money Accessed money from a bank account 27

Personal_Help Tried to get help from friends and/or 26 relatives

Rec Recreational activity (e.g. listen to music, 17 watched tv, played games, crafts...)

$\begin{array}{lll}\text { Drinking_Est } & \text { Drinking establishment or activity (e.g. } & 12\end{array}$ night out, party, nightclub, bars, or pubs)

Attended_Police Attended, on route to Police office/station 11 or contacted police.

Unfortunate Unfortunate incident (e.g. missed bus, ran 6 out of petrol, car crash, phone ran out of battery....)

Holiday Went on holiday/day out 3

Table 3: Dominant theme and demographic characteristics

\begin{tabular}{llllll}
\hline & & Dysfunctional & Escape & Unintentional & Chi square p \\
\hline Gender & Male & $46(28 \%)$ & $30(18 \%)$ & $91(55 \%)$ & n.s \\
& Female & $21(24 \%)$ & $9(10 \%)$ & $58(66 \%)$ & \\
Ethnicity & White & $44(24 \%)$ & $26(14 \%)$ & $114(62 \%)$ & n.s \\
& Non white & $15(32 \%)$ & $7(15 \%)$ & $25(53 \%)$ & \\
Age & $18-30$ & $18(18 \%)$ & $6(6 \%)$ & $75(76 \%)$ & $<.001$ \\
& $31-60$ & $37(29 \%)$ & $28(22 \%)$ & $64(50 \%)$ & \\
& Older 60 & $12(44 \%)$ & $5(19 \%)$ & $10(37 \%)$ & \\
Occupation & Employed & $19(23 \%)$ & $21(26 \%)$ & $42(51 \%)$ & .003 \\
& Not employed & $41(28 \%)$ & $13(9 \%)$ & $92(63 \%)$ & \\
Mental & Yes & $47(33 \%)$ & $20(14 \%)$ & $74(53 \%)$ & .017 \\
health & & & & & \\
disorder & & & & & \\
& No & $20(18 \%)$ & $19(17 \%)$ & $75(66 \%)$ & \\
Risk & High & $32(35 \%)$ & $25(27 \%)$ & $35(38 \%)$ & \\
& Medium & $18(20 \%)$ & $11(12 \%)$ & $60(67 \%)$ & \\
& Low & $17(23 \%)$ & $3(4 \%)$ & $54(73 \%)$ &
\end{tabular}

